\title{
Research Square \\ The Impact of Colchicine on The COVID-19 Patients; A Clinical Trial Study
}

\section{Farhad Salehzadeh}

ARUMS: Ardebil University of Medical Sciences

\section{Farhad Pourfarzi}

ARUMS: Ardebil University of Medical Sciences

Sobhan Ataei ( $\sim$ Sobhan13295@gmail.com )

ARUMS: Ardebil University of Medical Sciences

\section{Research article}

Keywords: Covid-19, Colchicine, Hydroxychloroquine

Posted Date: September 21st, 2020

DOl: https://doi.org/10.21203/rs.3.rs-69374/v1

License: (1) This work is licensed under a Creative Commons Attribution 4.0 International License.

Read Full License 


\section{Abstract}

Background: Severe acute respiratory syndrome COVID-19 infection has evolved into a global pandemic. This study has been designed to evaluate colchicine anti-inflammatory effect on the symptoms course, duration of hospitalization, morbidity and mortality rate, of COVID-19 patients.

Methods: In this prospective, open-label, randomized and double blind clinical trial, 100 patients hospitalized with COVID-19 were randomized in a 1:1 allocation from May 21 to June 20, 2020, to either standard medical treatment (Hydroxychloroquine) or colchicine with standard medical treatment. The study took place in Imam Reza hospital of Ardabil city in Iran, with trial registration ID: 47707 (irct.ir). Colchicine group were received $1 \mathrm{mg}$ tablet of colchicine daily alongside the Hydroxychloroquine for 6 days. Primary end points were (1) Length of hospitalization; (2) symptoms and (3) Co-existed disease. Secondary end points were examined 2 weeks after discharge and included (1) mortality and morbidity; (2) re-admission and (3) symptoms.

Results: Overall, 100 patients (59 [59\%] female; median age, 56 years) fulfilled the admission criteria and were included and randomized at 2 clinical groups. There was no significant difference between the two groups in terms of age and sex. Two groups were not significantly different in terms of underlying diseases and various clinical and para clinical findings although there were not any different during Postdischarge follow-up except for duration of fever $(P<0.05)$. Comparing two groups showed significantly different only in the duration of hospitalized $(P<0.05)$. Although in colchicine group dyspnea was improved more rapid than the placebo group, but it was not meaningful.

Conclusion: Colchicine can be effective in reducing systemic symptoms of COVID-19 by inhibiting inflammatory biomarkers.

Current Controlled prospective Trials registration ID that has been approved by ICMJE and WHO ICTRP registry is IRCT20200418047126N1, and the date of registration is 2020-05-14.

\section{Background}

In late 2019, several cases of an acute respiratory illness (now known as the new coronavirus or COVID19) were reported in Wuhan, China (1-3).

The coronavirus has spread rapidly to all over the world. As of August 16, 2020, a total of about 21.2 million people infected by the virus and caused 761000 deaths all over the world (4).

There is no clear information on the pathophysiology of the disease. Many studies have suggested that an overreaction of the immune system by virus can cause the complicated features of disease (5). Cytokine storm syndrome is the sever immune reaction that may cause a severe tissue response in these patients (6). 
NLRP3, an inflammasome compartment activates IL-18, IL-1 $\beta$ and IL-6 (7). Excessive synthesis of IL-6 against infection leads to an acute systemic inflammatory reaction known as cytokine storm (8).

IL- 6 which plays the main role in cytokine storm, is produced by activated leukocytes causing excretion of several other cytokines subsequently. On the other hand the production of these cytokines is mainly triggered in order to develop an inflammation to suppress the infection (9). Considering the role of inflammation in both exacerbation and suppression of the disease it can be hypothesized that altered mechanisms of innate immunity pathway as a main role in IL-6 production may result in different clinical features of the disease.

Acute lung injury and even ARDS are common outcomes of cytokine storm in lung alveolus (10).

Colchicine is a drug that is widely used to treat and prevent acute gout attacks, and other crystal arthropathies, Familial Mediterranean Fever (FMF), and systemic vasculitis such as Behcet disease. In addition, it has been shown that colchicine is an important drug in inflammatory diseases due to its widespread anti-inflammatory effects particularly by stabilizing of polymorph nuclear cells (PMN) (11).

Colchicine affects NLRP3 and prevents the activation of IL-18, IL-1 $\beta$ and IL-6 (7), and appears to play a significant role in reducing and controlling of the cytokines storm

According to its anti-inflammatory effects, it seems that Colchicine may have a significant effect on reducing the symptoms, course and mortality rate caused by the new coronavirus disease. Therefore, this double blind clinical trial study has been conducted to evaluate the treatment effect of this drug on the symptoms, duration of hospitalization, morbidity and mortality rate, of COVID-19 patients.

\section{Methods}

\section{Patients and data.}

Patient recruitment started on May 21, 2020, and was terminated on June 20, 2020 in Imam Reza hospital in Ardabil city. A phase 3, randomized, double blind clinical trial with control group was conducted 100 adult patients with COVID-19. Patients met the inclusion criteria, which were defined as follows: Pulmonary involvement seen in CT-Scan compatible with COVID-19 and Positive PCR of COVID19. Exclusion criteria were defined as follows: sensitivity to any medications of regimens, renal failure, heart failure, pregnancy, participating in another clinical study and refusal to participate in the study before or during the follow-up period.

\section{Treatment regimens}

This is prospective, randomized clinical trial, open labeled, controlled group study. Patients were randomized in 1:1 allocation in two groups (group-A and group-B) which contains 50 patients. Patients of group-A will be treated under Hydroxychloroquine as a health care system guideline treatment and colchicine regime while in group-B patients was treated with Hydroxychloroquine alone plus placebo. 
Each group contains 50 people. Individuals of intervention group (A) were received $1 \mathrm{mg}$ tablet of colchicine daily alongside the Hydroxychloroquine for 6 days. The participants of the placebo group were received a similar tablet without therapeutic effects alongside the Hydroxychloroquine for 6 days. Hydroxychloroquine was a drug that is included in our health care protocol and all of patients in this study were received same treatment such as Azithromycin in their therapy period.

\section{Study designs, evaluations and endpoints}

Clinical assessments were conducted after the first dose of medication and their follow-up 2 weeks after discharge. Assessments will be compared with the first day of admission. This study adheres to CONSORT guidelines and include a completed CONSORT checklist.

All patients of the study filled confirmed consent form.

\section{Statistical Analysis}

In this study, SPSS statistical analysis software version 25 was used to analyze the data. The data were first expressed using the frequency command (number, percentage, mean) and then using independent Ttest and chi-square test, the relationship between them was examined and the results were presented in tables. To evaluate the significance of the deficiency, foundation was used, which was considered significant less than 0.05 . Current Controlled prospective Trials registration ID that has been approved by ICMJE and WHO ICTRP registry is IRCT20200418047126N1, and the date of registration is 2020-05-14.

\section{Results}

Overall, 100 patients (59 [59\%] female; median age, 56 years) fulfilled the admission criteria and were included and randomized at 2 clinical groups. Their baseline characteristics are summarized in Table 1. 
Table 1

Baseline Characteristics of patients in two groups

\begin{tabular}{|c|c|c|}
\hline \multirow[t]{2}{*}{ Characteristic } & \multicolumn{2}{|l|}{ No. (\%) } \\
\hline & Colchicine group $(n=50)$ & Placebo group $(n=50)$ \\
\hline Male & $19(38)$ & $22(44)$ \\
\hline Female & $31(62)$ & $28(56)$ \\
\hline Age (year) & 56.56 & 55.56 \\
\hline Time from suffering to & 6.28 & 8.12 \\
\hline \multicolumn{3}{|l|}{ Enrollment (day) } \\
\hline \multicolumn{3}{|l|}{ Co-existed disease: } \\
\hline Diabetes Mellitus & $5(10)$ & $6(12)$ \\
\hline Ischemic Heart Disease & $6(12)$ & $9(18)$ \\
\hline Hypertension & $3(6)$ & $8(16)$ \\
\hline Cancer/Neoplastic Disorder & $1(2)$ & $1(2)$ \\
\hline COPD & $0(0)$ & $4(8)$ \\
\hline Renal failure & $4(8)$ & $1(2)$ \\
\hline Hypothyroidism & $1(2)$ & $1(2($ \\
\hline \multicolumn{3}{|l|}{ Symptoms: } \\
\hline Fever & $34(68)$ & $39(78)$ \\
\hline Myalgia & 18(36) & $22(44)$ \\
\hline Cough & $30(60)$ & $33(66)$ \\
\hline Dyspnea & $21(42)$ & $18(36)$ \\
\hline Vomiting & $6(12)$ & $2(4)$ \\
\hline Nausea & $8(16)$ & $9(18)$ \\
\hline Sweating & $4(8)$ & $9(18)$ \\
\hline Headache & $7(14)$ & $9(18)$ \\
\hline \multicolumn{3}{|l|}{ Laboratory: } \\
\hline $\begin{array}{l}\text { White blood cell count, } \\
\text { mean, } / \mu \mathrm{L}\end{array}$ & 6544 & 6486 \\
\hline
\end{tabular}




\begin{tabular}{|cll|}
\hline Characteristic & No. $(\%)$ & \\
\cline { 2 - 3 } & Colchicine group $(\mathbf{n}=50)$ & Placebo group $(\mathbf{n}=\mathbf{5 0})$ \\
\hline Neutrophil count & $4916.14 / \mu \mathrm{L}$ & $4894.66 / \mu \mathrm{L}$ \\
\hline Lymphocyte count & $1136.40 / \mu \mathrm{L}$ & $1255.62 / \mu \mathrm{L}$ \\
\hline Eosinophil count & $189.78 / \mu \mathrm{L}$ & $269.82 / \mu \mathrm{L}$ \\
\hline Monocyte count & $100.12 / \mu \mathrm{L}$ & $90.80 / \mu \mathrm{L}$ \\
\hline Hemoglobin, mean, g/dL & 12.81 & 12.91 \\
\hline Platelet, mean, $\times 10^{3} / \mu \mathrm{L}$ & 192 & 200 \\
\hline Creatinine, mean, gr/dL & 1.23 & 1.06 \\
\hline ESR, mean, mm/hr & 37.73 & 43.13 \\
\hline AST, mean, $/ \mathrm{L}$ & 35.25 & 114.22 \\
\hline ALT, mean, /L & 40.48 & 115.28 \\
\hline Alk.p, mean, /L & 179.63 & 185.51 \\
\hline PT, mean, /s & 14.38 & 15.33 \\
\hline PTT, mean, /s & 35.32 & 33.30 \\
\hline INR, mean & 1.06 & 1.21 \\
\hline
\end{tabular}

There is no significant difference between the two groups in terms of age and sex. Two groups are not significantly different in terms of underlying diseases and various clinical and para clinical findings although there were not any different during post-discharge follow-up except for duration of fever $(\mathrm{P}<$ 0.05). Comparing two groups for quantitative variables with t-test shows significantly different only in the duration of hospitalized $(P<0.05)$. (Table 2). Although in colchicine group dyspnea was improved more rapid than the placebo group, but it was not meaningful. 
Table 2

Post discharge findings of two groups

\begin{tabular}{|llll|}
\hline Characteristic & No. $(\%)$ & P Value \\
\cline { 2 - 4 } & Colchicine group (n=50) & Placebo group (n= 50) \\
\hline Duration of hospitalized, /day & 6.28 & 8.12 & 0.001 \\
\hline Diabetes Mellitus & $5(10)$ & $6(12)$ & 0.749 \\
\hline Ischemic Heart Disease & $6(12)$ & $9(18)$ & 0.401 \\
\hline Hypertension & $3(6)$ & $8(16)$ & 0.110 \\
\hline Cancer/Neoplastic Disorder & $1(2)$ & $1(2)$ & 1 \\
\hline COPD & $4(8)$ & $1(2)$ & 0.169 \\
\hline Renal failure & $1(2)$ & $1(2($ & 1 \\
\hline Fever & $1(2)$ & $11(22)$ & 0.02 \\
\hline Myalgia & $2(4)$ & $4(8)$ & 0.400 \\
\hline Cough & $2(4)$ & $2(4)$ & 1 \\
\hline Dyspnea & $3(6)$ & $6(12)$ & 0.295 \\
\hline Vomiting & $0(0)$ & $2(4)$ & 0.153 \\
\hline Nausea & $4(8)$ & $3(6)$ & 0.695 \\
\hline Sweating & $0(0)$ & $0(0)$ & - \\
\hline Headache & $1(2)$ & $3(6)$ & 0.307 \\
\hline
\end{tabular}

\section{Discussion}

There is no specific treatment recommended for COVID-19, and no vaccine is currently available. The only treatment is symptomatic, and oxygen therapy that is useful for respiratory impairment. In points with respiratory failure non-invasive and invasive mechanical ventilation may be necessary (12).

Convalescent plasma and or immunoglobulin have been used as alternative resort to improve the survival rate of patients with COVID-19 whose condition continued to deteriorate despite treatment with methylprednisolone pulse therapy (13).

Epidemiologic studies have been shown 5\% of infections with COVID-19 characterized by acute respiratory distress syndrome (ARDS), requiring mechanical ventilation. Concerning ARDS treatment; it seems plausible to speculate that the anti-IL6R mAb plays a protective role if given at the time of overly elevated immune response to the virus, thus preventing "anaphylactic toxicity". Such extreme cytokine 
reaction is accompanied by infiltration of inflammatory monocytes/macrophages (IMM) into the lung and elevated production of the pro-inflammatory cytokines (IL-1, IL-6, IL-8, CXCL-10, and MCP-1) (14).

Colchicine has been shown to limit IL-1b production as a response to various NLRP3 inflammasome inducers in a dose-dependent form. For example, in the setting of acute coronary syndrome, colchicine was effective in suppressing interleukin IL-1b, IL-18 and IL-6, which was attributed to inflammasome inhibition $(15,16)$.

Chloroquine and hydroxychloroquine have been found to be efficient on SARS-CoV-2, and reported to be efficient in Chinese COV-19 patients (17).

Our clinical trial study included 100 patients in two randomized groups. The two groups did not differ significantly on underlying diseases. In terms of symptoms, during the follow-up after discharge; fever was significantly lower in the group receiving colchicine $(P=0.02)$. Although the hospitalized period is significantly different between groups $(P=0.001)$ and was less in colchicine group. None of the patients died or were readmitted.

In Spyridon et al study (18), about the colchicine effect on cardiac and inflammatory biomarkers in COVID-19 admitted patients. Mean (SD) event-free survival time was $18.6(0.83)$ days the in the control group vs $20.7(0.31)$ in the colchicine group ( $\log$ rank $P=0.03)$. In our study, the level of fever in the colchicine group was significantly lower than in the placebo group $(P=0.02)$. Moreover in colchicine group dyspnea was subsided more rapidly than the placebo group.

In Mansouri et al study (19); they described the case of a 42 years old, healthy patient with Covid-19 who despite improvement in his respiratory symptoms developed a mild to moderate cytokine release syndrome (CRS) and an associated mono articular gout flare. Since the patient refused admission to the hospital and had stable vital signs, they treated him with a safe anti-inflammatory and nonimmunosuppressive therapy. To hit two birds with one stone, they considered colchicine, as it has systemic anti-inflammatory effects and is also effective in gout flare. Unexpectedly, 48 hours after treatment, not only did his ongoing fever and toe pain disappear, he also had significant improvements in his general state of health and all his inflammatory markers including fibrinogen, ferritin, D-dimer, and IL-6 levels normalized.

Due to the inflammatory basis in the COVID-19 that includes cytokine storm syndrome because of excessive synthesis of IL- 6 against infection and also due to the anti-inflammatory effects of colchicine on the innate immune system by stabilizing of PMNs; Colchicine seems to be effective in improving systemic COVID-19 symptoms such as fever, which is undoubtedly due to inflammatory biomarkers such as IL-6 and TNF (tumor-necrosis factor). Inhibition of these biomarkers is certainly effective in preventing acute respiratory syndrome and cytokine storm suppression the progressiveness of disease which is the most dangerous sign in COVID-19 $(6,8)$. However; it is necessary to confirm this idea with further studies.

\section{Limitations}


This study was performed only on the clinical aspects and symptoms of patients and the changes in biomarkers were not evaluated. This study was performed in only non-ICU patients to evaluate their course of disease.

On the other hand, the use of hydroxychloroquine in patients due to the health ministry guideline may have a combination medicinal side effects.

\section{Conclusion}

Colchicine can be effective in reducing systemic symptoms of COVID-19 by inhibiting inflammatory biomarkers.

\section{Abbreviations}

COVID-19: Coronavirus Disease 2019

IL: Interleukin

ARDS: Acute respiratory distress syndrome

ICU: Intensive care unit

\section{Declarations}

\section{Ethics approval and consent to participate:}

This study has been supported by Ardabil University of Medical Sciences (ARUMS) and approved by Ministry of Health committee of medicine under the number: Custom Trial registration ID is: 47707 (irct.ir). The written confirmed consent obtained from all participants.

\section{Consent for publication:}

Authors taken written informed consent, to do of this work.

\section{Competing interests:}

The authors declare that they have no competing interests.

\section{Conflict of Interest:}

There is not any conflict of interest in this study.

\section{Funding:}

Authors declare any private funding in this study. 


\section{Authors Contributions:}

All authors have read and approved the manuscript and they contribute as

FP. Worked on epidemiologic aspects of study, FS designed the study and wrote draft copy, SA collected all data and wrote final copy.

Acknowledgements: The authors would like to acknowledge the Faculty of medicine, Ardabil University of medical sciences, and Imam Reza hospital for their support and contribution to this study.

\section{Availability of data and material:}

Not applicable

\section{References}

1. Lu H, Stratton CW, Tang YW. Outbreak of pneumonia of unknown etiology in Wuhan China: the mystery and the miracle [published January 16, 2020]. J Med Virol. 2020.

doi:10.1002/jmv.25678PubMedGoogle Scholar

2. Hui DS, I Azhar E, Madani TA, et al. The continuing 2019-nCoV epidemic threat of novel coronaviruses to global health: the latest 2019 novel corona cytokine virus outbreak in Wuhan, China [published January 14, 2020]. Int J Infect Dis. 2020; 91:264-266.

3. Wuhan Municipal Health Commission. Report of clustering pneumonia of unknown etiology in Wuhan City. Published December 31, 2019. Accessed January 31, 2020. http://wjw.wuhan.gov.cn/front/web/showDetail/2019123108989.

4. World Health Organization. Coronavirus disease (COVID-19): situation report. Accessed August 16, 2020. https://www.who.int/docs/default-source/coronaviruse/situation-reports/20200817-weeklyepi-update-1.pdf?sfvrsn=b6d49a76_4

5. Vardhana SA, Wolchok JD. The many faces of the anti-COVID immune response. Journal of Experimental Medicine. 2020;217(6).

6. Mehta P, McAuley DF, Brown M, Sanchez E, Tattersall RS, Manson JJ. COVID-19: consider cytokine storm syndromes and immunosuppression. The Lancet. 2020;395(10229):1033-4.

7. Vitiello A, Ferrara F, Pelliccia C, Granata G, La Porta R. Cytokine storm and colchicine potential role fighting SARS-CoV-2 pneumonia. Ital J Med [Internet]. 2020Apr.29 [cited 2020May22];00. Available from: https://italjmed.org/index.php/ijm/article/view/itjm.2020.1284

8. Tanaka T, Narazaki M, Kishimoto T. Immunotherapeutic implications of IL-6 blockade for cytokine storm. Immunotherapy. 2016;8(8):959-970. doi:10.2217/imt-2016-0020.

9. Zhang C, Wu Z, Li J-W, Zhao H, Wang G-Q. The cytokine release syndrome (CRS) of severe COVID-19 and Interleukin-6 receptor (IL-6R) antagonist Tocilizumab may be the key to reduce the mortality. International journal of antimicrobial agents. 2020:105954. 
10. Jennifer R. Tisoncik, Marcus J. Korth, Cameron P. Simmons, Jeremy Farrar, Thomas R. Martin, Michael G. Katze. Microbiology and Molecular Biology Reviews Mar 2012, 76 (1) 16-32; DOI: 10.1128/MMBR.05015-11.

11. Anastasia Slobodnick, Binita Shah, Svetlana Krasnokutsky, Michael H. Pillinger, Update on colchicine, 2017, Rheumatology, Volume 57, Issue suppl_1, 1 January 2018, Pages i4-i11, https://doi.org/10.1093/rheumatology/kex453.

12. Cascella M, Rajnik M, Cuomo A, Dulebohn SC, Di Napoli R. Features, Evaluation and Treatment Coronavirus (COVID-19). In: StatPearls. Treasure Island (FL): StatPearls Publishing; 2020.

13. Chen L, Xiong J, Bao L, Shi Y. Convalescent plasma as a potential therapy for COVID-19. Lancet Infect Dis. 2020;20(4):398-400. doi:10.1016/S1473-3099(20)30141-9.

14. Buonaguro, F.M., Puzanov, I. \& Ascierto, P.A. Anti-IL6R role in treatment of COVID-19-related ARDS. J Transl Med 18, 165 (2020). https://doi.org/10.1186/s12967-020-02333-9.

15. Martínez GJ, Robertson S, Barraclough J, Xia Q, Mallat Z, Bursill C et al. Colchicine Acutely Suppresses Local Cardiac Production of Inflammatory Cytokines in Patients With an Acute Coronary Syndrome. Journal of the American Heart Association. 2015 Aug 24;4(8):e002128. https://doi.org/10.1161/JAHA.115.002128.

16. Stacy Robertson, Gonzalo J. Martínez, Cloe A. Payet, Jennifer Y. Barraclough, David S. Celermajer, Christina Bursill, Sanjay Patel; Colchicine therapy in acute coronary syndrome patients acts on caspase-1 to suppress NLRP3 inflammasome monocyte activation. Clin Sci (Lond) 1 July 2016; 130 (14): 1237-1246. doi: https://doi.org/10.1042/CS20160090.

17. Gautret P, Lagier JC, Parola P, et al. Hydroxychloroquine and azithromycin as a treatment of COVID19: results of an open-label non-randomized clinical trial [published online ahead of print, 2020 Mar 20]. Int J Antimicrob Agents. 2020;105949. doi:10.1016/j.ijantimicag.2020.105949.

18. Deftereos SG, Giannopoulos G, Vrachatis DA, et al. Effect of Colchicine vs Standard Care on Cardiac and Inflammatory Biomarkers and Clinical Outcomes in Patients Hospitalized With Coronavirus Disease 2019: The GRECCO-19 Randomized Clinical Trial. JAMA Netw Open. 2020;3(6):e2013136. doi:10.1001/jamanetworkopen.2020.13136.

19. Nahal Mansouri, Majid Marjani, Payam Tabarsi, Christophe von Garnier \& Davood Mansouri (2020) Successful Treatment of Covid-19 Associated Cytokine Release Syndrome with Colchicine. A Case Report and Review of Literature, Immunological Investigations, DOI: 10.1080/08820139.2020.1789655.

\section{Supplementary Files}

This is a list of supplementary files associated with this preprint. Click to download.

- CONSORT2010Checklist1.doc 